\title{
Contribution to predefinition of the geometric features and their processing sequences in order to create a CAPP model
}

\author{
Iaroslav Francisc Pișec* and Florin Sandu Blaga \\ University of Oradea, Department of Industrial Engineering, Universităţii 1, Oradea, Romania
}

\begin{abstract}
In order to create a Computer Aided Process Planning (CAPP) model for a family of parts, it is necessary to establish a database containing the geometric features specific to that family. In the car body production line industry, several families of parts can be determined each having specific geometric features. The parts that are part of a family of parts must be designed in such a way that at the end, they contain those specific features. The next step in the implementation of the CAPP model, is to create a database for processing sequences associated with geometric features. Combining these two databases, the database of geometric features and the processing sequences, the result is the NC program of the designed part. This study presents the creation of these two databases and the link between them.
\end{abstract}

\section{Introduction}

With increase in global competition in manufacturing, there is a need to automate this process to reduce the lead time, improve quality, productivity and product development life cycles. In this regard, Computer Aided Process Planning (CAPP) was introduced as a bridge between CAD and CAM systems that were developed independently [1].

CAPP is the application of computer to assist the human process planer in the process planning function. In its lowest form it will reduce the time and effort required to prepare process plans and provide more consistent process plan. In its most advanced state, it will provide the automated interface between $\mathrm{CAD}$ and $\mathrm{CAM}$ and in the process achieve the complete integration with in CAD/CAM.

In the last few decades, many CAPP systems were developed. CAPP systems can be classified depending on whether they are feature-based, knowledge-based, agent-based, or whether they use neural networks, genetic algorithms, fuzzy logic or Petri Nets. Among these, feature-based technologies have long been used in CAD/CAM integrations, as most CAM systems identify features as input data [1].

Babic et al. [2] classified feature-based technologies into three types: design by features (DBF), interactive form feature recognition and automated feature recognition. Parts are designed using features from libraries in the DBF methodology.

\footnotetext{
${ }^{*}$ Corresponding author: pisec.iaroslav@gmail.com
} 
Planning for machining sequence can be considered as one of the most important functions of manufacturing process planning [3]. Weight and material of the raw part are determined by the functional requirement of plan. The next logical step in process planning is to determine the appropriate types of processing operations and their sequences to transform the features, dimensions and tolerances of a part from the raw to the finished state.

\section{Description of a family of part from a car body production line}

Within a car body production line, there are various systems and devices whose role is to create the bodywork at the end of the line. These systems are standards, customized or dedicated. Dedicated systems are specific to each stage of assembly and welding of the array assemblies. They mostly contain unique pieces, made only once for the line, for the system they are part of.

\subsection{Classification of parts in family of parts}

Designed and manufactured parts can be divided into two major categories:

- complex parts produced in a small number;

- simple parts produced in a large number.

The complex parts combine all those parts whose machining is lasting and costly, the material of the semi-finished product is special and these parts are in a small number within the bodywork line.

Single parts count for about $85 \%$ of the total track parts. These can be framed in several family of parts. The main families of parts that can be formed according to the types of parts existing in a production line are:

- "PIN" family of parts;

- "LOCATOR BLOCK" family of parts;

- "L BLOCK" family of parts;

- "PLATE" family of parts;

- "BLADE" family of parts;

- "WELDMENT SIMPLE" family of parts.

\subsection{Description of the parts from the "PIN" family of parts}

The PIN is a component part of the vehicle body fastener and assembly device used to centre the element and to cancel one or two degrees of freedom respectively. Figure 1 shows two types of pins. These centering pins, together with all pins from the fastener and assembly devices, form the "PIN" family of parts.

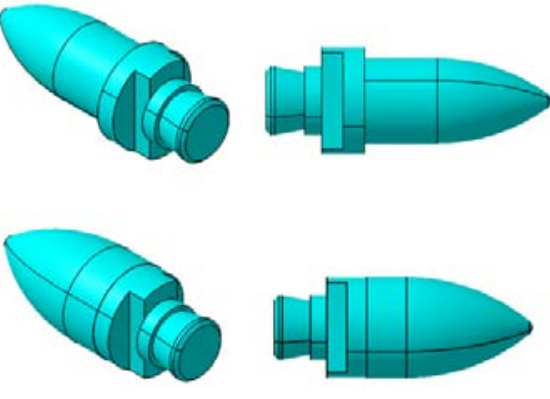

Fig. 1. Parts components of "PIN" family of parts.

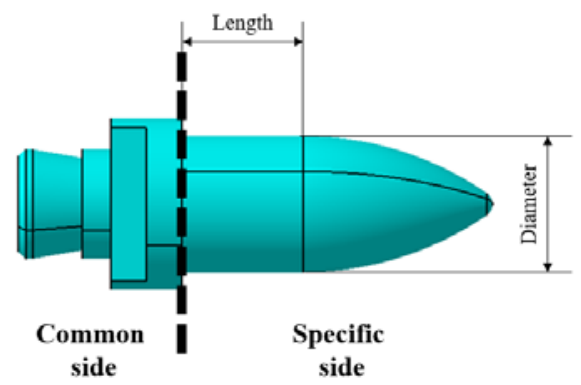

Fig. 2. Delimitation of the common side and the specific side of the PIN. 
The PIN consists of two sides: the common side found at all the pins, and the specific side, Figure 2. So, to produce a PIN it can be said that it is necessary to process two different side. The common side represent the shape and dimensions of the PIN fixing system. The specific side is determined by the diameter of the hole in the body element and the height at which it is located. It follows that the main characteristics of the specific side are the diameter of the PIN and its active length,

In this paper it is presents how to create a CAPP (Computer Aided Process Planning) model for the "PIN" family of parts. First of all, it is intended to predefine the geometric entities and then to predefine the processing sequences specific for each component side of the PIN, according to specific parameters. These will be stored in databases from where can be easy accessed later.

\section{Geometric features databases}

The first step in creating a database is to analyse the data and to create a conceptual scheme of these data. At this stage the nature and usage of the data are analysed. Data that will need to be stored and processed is identified. Data analysis is sometimes a difficult process that takes a lot of time, but it is an absolutely obligatory stage. These databases are updated each time when a new object is introduced, updating manually by the operator.

The database is an organized collection of graphical and non-graphic data stored on a memory card in the computer. The role of a database is to collect and maintain data in a central storage area, so it is available to operators and policy makers.

The advantages of a centralized database are:

- applying standards;

- elimination of redundancy;

- applying security restrictions;

- compensation of conflicts;

- maintaining integrity.

Although there are several types of databases, the easiest to create and then to access is the object-oriented database.

The object-oriented database requires object-oriented access and manipulation. The object-oriented model must be able to store all relevant information. Object-oriented database models include relationships between entities, complex object representations, molecular object representation, and abstract data.

\subsection{Predefinition of the geometric features}

The procedure for organizing the database for the construction of a real three-dimensional object starts with the analysis of the part model. As shown above, the PIN is divided into two component sides. In Figure 3 is presented the PIN object-oriented database.

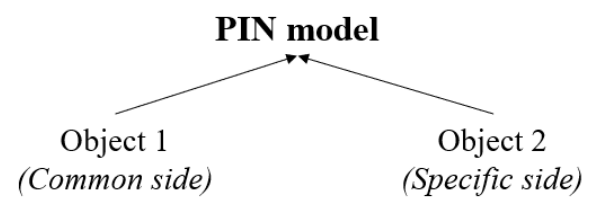

Fig. 3. Object-oriented database for the pin CAD model.

The geometric features are defined interactively. This often leads to the identification of elements belonging to a geometric entity for the analysed model. It can start with a complete geometric model and then define the specific geometric entities that are saved in a standard database. 
Forwards, is presented the way how to define the geometric entities for each side of the PIN. For the common PIN side are showed in Figure 4, the specific geometric entities which will form the Object 1.

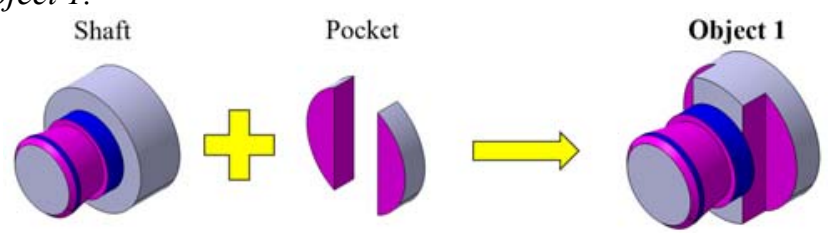

Fig. 4. Geometric entities which form the Object 1, common PIN side.

For the specific PIN side are showed in Figure 5, the specific geometric entities which will form the Object 2.

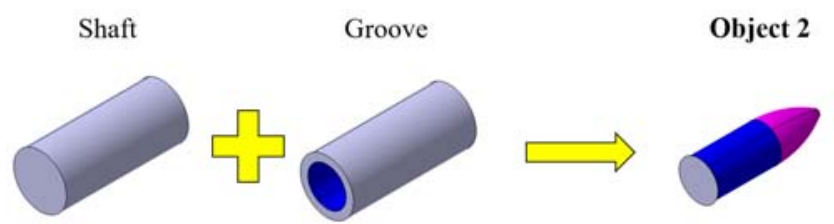

Fig. 5. Geometric entities which form the Object 2, specific PIN side.

\subsection{Geometric entities database creation}

To create a geometric entities database is necessary to use a CAD design software. In our case, is used the CATIA V5 software from Dassault Systemes ${ }^{\circledR}$. This software has a knowledge set tool named Power Copy. A power copy is basically a copy of a group of features and it can have various inputs defined to modify it. It allows for an item to be reused in multiple situations yet keeping the same standard methodology of creation.

In Figure 6 is presented the power copy definition of the common PIN side. In tab Definition are selected the components which are necessary to instantiate, and these are the shaft and pocket. Second step is to define the input which is YZ plane.
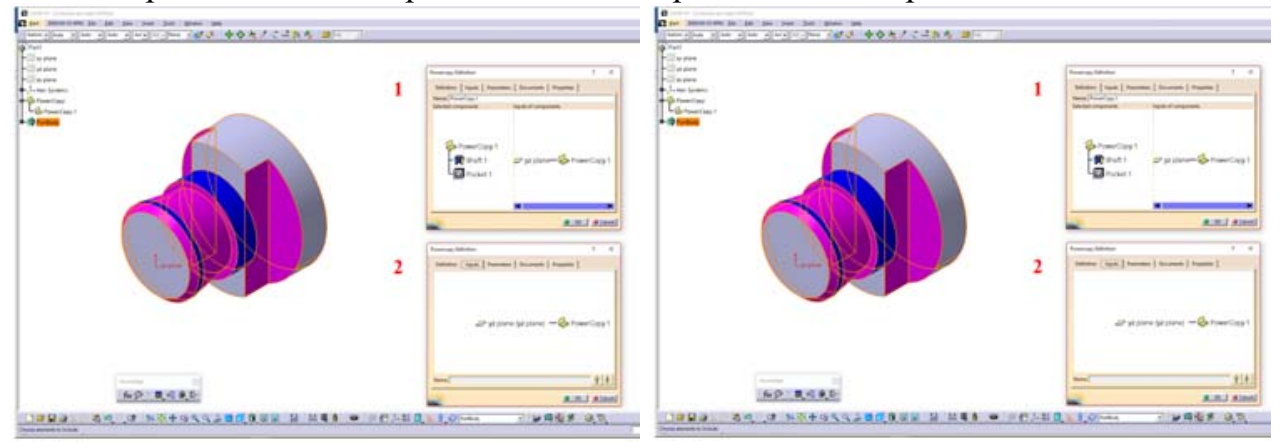

Fig. 6. Power copy definition for common pin side. Fig. 7. Power copy definition for specific pin side.

After power copy definition, the 3D model is saved as CATIA Part in a specific location from where later can be accessed. In this location will be saved all the power copies and together will determinate the geometric feature database.

In Figure 7 is presented the power copy definition of the specific PIN side. In tab Definition is selected the shaft as instantiated component. Second step is to define YZ plane as input and third step is publishing those parameters which are variable, diameter and length. 


\subsection{Designing the pin using power copies of geometric entities}

In Figure 8 is presented how is designed a PIN using the power copies of geometric entities from database. First is added the common PIN side and then the specific side where is defined the PIN diameter and the length.

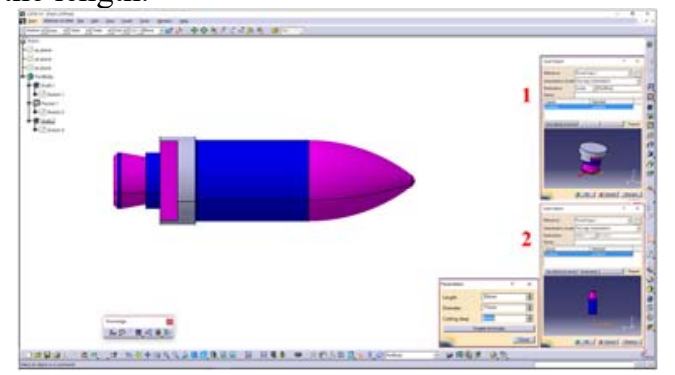

Fig. 8. Designing the PIN.

\section{Processing sequences databases}

The next step in the implementation of the CAPP model, is to create a database for processing sequences associated with geometric features. Processing sequences are used to specify the sequence of machining operations that must be performed on PIN during manufacturing process. Definition of this sequences is made by a person which have a good understanding of machining practice and is using a Computer Aided Manufacturing software.

In Figure 9 is presented the way how are defined the processing sequences. Also, for these, is used the component sides of the PIN which determinate the creation of two processing sequences sets: one set for common PIN side and another for specific PIN side.

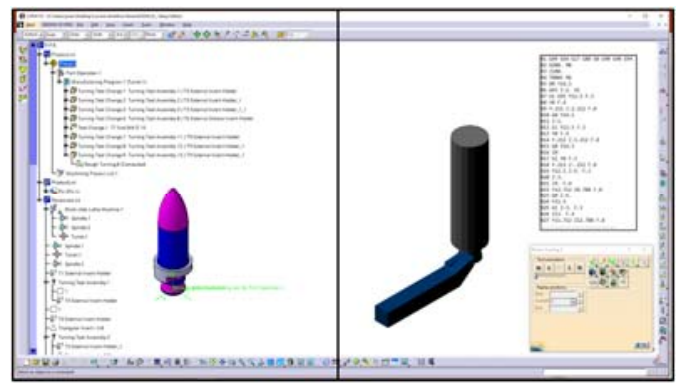

Fig. 9. Definition and simulation of tool paths; NC code for PIN.

The processing sequences are saved in an EXCEL file from where will be accessed to create the NC code for PIN manufacturing. The processing sequences for the common PIN side will be always the same but the processing sequences for the specific PIN side, will be variable. For this, in the EXCEL file, the NC code has few variables as show in Figure 10.

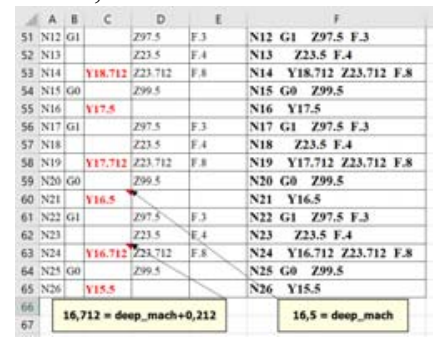

Fig. 10. Variables from $\mathrm{NC}$ code. 


\section{NC program generation of the designed part}

After designing the PIN, using a macro, is generate a EXCEL file saved in the same location with $3 \mathrm{D}$ model. This file is containing the information about the PIN and the diameter and the length of the specific pin side. After opening the CAPP module interface, is selected the "PIN" family of parts and this file is loaded into. The program is calculating the stock size for the PIN. Is important to have the stock sizes because from these, the CAPP module is starting to generate the processing sequences. In Figure 11 are presented the dimensions which are defining the stock sizes for PIN.
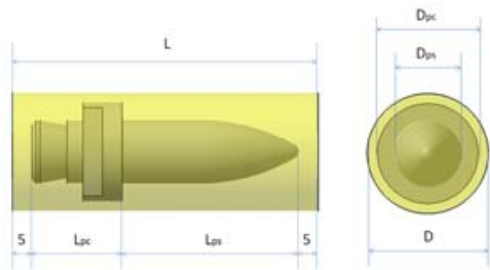

Fig. 11. Defining the stock workpiece dimensions.

Executing the button "Generate NC program", the CAPP module is replacing all variables from a processing sequences template with the data from input EXCEL file. The result is a $\mathrm{NC}$ code saved in a user define format and location.

\section{Conclusions}

This paper presents a roughly mode how a CAPP system can be define using the geometric features and processing sequences databases. The system is configured for a specific family of part, more exactly for the "PIN" family of parts. The geometric features database contains the user defined features, some constant other variable. The processing sequences database is an EXCEL file, where the technologist fills the NC code. This code contains variables which in final step, will be replaced by exact values. Replacing is started with a macro which is using information from the post-designed generated EXCEL file for the designed PIN.

With the CAPP system, result less machining time for manufacturing and less human interference. Starting with design and finishing with the finished part, it can be said that the actual time is reduced with $60-70 \%$.

\section{References}

1. D.M. Dierich, B.K. Jayanthi, A. Datar, S. Joshi, Institute of Ind. Eng., 2138-2144 (2014)

2. B. Babic, N. Nesic and Z. Miljkovic, Computers in Industry 59, 4, 321-337, (2008)

3. B.R. Borkar, Optimization of Machining Time using Feature Based Process Planning, 5th International \& 26th All India Manufacturing Technology, Design and Research Conference, (2014)

4. V. Allada, S. Anand, Inter. Journal of Prod. Res. 34, 7, (1996)

5. H. Zhang, L. Alting, Computerized manufacturing process planning systems, Springer Netherlands, 1, (1994)

6. C.V. Doicin, Contributions regarding the Computer Aided Process Planning of Shafts from the Machine Building Industry, PhD Thesis, (2001) 